\title{
Parents' accounts of their children's respiratory symptoms showed a range of interpretations
}

Young B, Fitch GE, Dixon-Woods M, et al. Parents' accounts of wheeze and asthma related symptoms: a qualitative study. Arch Dis Child 2002;87:131-4.

\section{QUESTION: How do parents recognise and make judgements about respiratory signs and symptoms in their young children?}

Design

Qualitative study using semistructured interviews.

\section{Setting}

Leicester, UK.

\section{Participants}

Parents of 19 children who were 1-6 years of age (eleven 1-3 y, eight 4-5 y; 12 boys) and were recruited from the cohort that had completed the Child Cohort Respiratory Symptoms Survey or attended one general practice in Leicester. The sample included parents who had never reported wheeze in their children and parents who had reported it, and also included families across a spectrum of social and cultural status. Interviews were done in the families' homes with the father alone $(n=2)$, the mother alone $(n=14)$, and the mother and father $(n=3)$.

\section{Methods}

The interviews were guided by a prompt list, audiotaped, and transcribed verbatim. Interview transcripts were analysed using the constant comparative method. Codes were applied, and the data were organised into thematic categories. Data were collected until theoretical saturation occurred.

\section{Main findings}

13 families reported previous or current history of wheeze in their children, and 6 families reported no wheeze. Parents described the sound of wheeze with such words as crackly, squeaky, strangled, gasping, rasp, rattle, lisp, animal sounding, and air noise. High pitched or whistling were the most common words used. Some parents used words to describe features that were felt rather than heard, such as strangled or gasping. Some also referred to changes in a child's appearance, chest movements, and vibrations felt when holding the child. When asked what the term "attacks of wheezing" meant, parents gave a range of interpretations from an episode with sudden onset to prolonged or severe episodes. Some parents considered every episode of wheeze as an attack.

Several parents had not witnessed shortness of breath in their children. Most described shortness of breath as a change in their child's breathing (labouring to breathe), appearance (change in complexion or looking "panicky" or "worn out"), and changes in behaviour (activity taking place at the time), with children having to stop or "slow down".
Parents distinguished among different types of cough and described 4 main features: dryness or wetness, depth, repetitiveness or persistence, and ticklishness. Several parents distinguished between an "asthma cough" and other types of cough.

Parents relied mostly on their own intimate knowledge of what was normal for their children in making judgments about their respiratory symptoms.

\section{Conclusions}

Parents showed variability in interpreting respiratory signs and symptoms in their young children. Many parents applied meanings to terms that differ from what is meant in clinical settings.
Source of funding: no external funding. Dr B Young, Department of Psychology, University of Hull, Hull, UK B.Young@hull.ac.uk
For correspondence:

\section{COMMENTARY}

Parents have an important role in assessing their children's symptoms, but care is required in phrasing the questions put to parents and interpreting the information they provide. It is known that such routinely used terms as "wheeze" can have different meanings for parents and professionals. ${ }^{1}$ In the study by Young et al, symptoms were interpreted in several ways by a group of parents of young children. Parents' assessments were based on observation of all aspects of their children rather than isolated symptoms. For example, they referred to appearance and other sensations as well as to the sound of breathing when describing "wheeze". Parents' assessments of symptoms drew on experiential knowledge of children and previous occurrences of symptoms. The absence of other symptoms was also considered in assessing whether or not a cough was indicative of asthma. Assessment of "shortness of breath" highlighted the difficulty of proxy assessment of symptoms that are experienced rather than seen. Parents referred to changes in breathing, appearance, and behaviour to describe shortness of breath. They were therefore describing the outward signs rather than the child's own experience of shortness of breath. This suggests that parents should be asked about observable signs of symptoms rather than internal feelings experienced by children.

Clinical implications of the study include the importance of recognising that parents' assessment of symptoms can be influenced by contextual factors, by a wider range of sensations than health professionals would use to assess some symptoms, and that parental assessment depends upon observation to describe young children's experience of symptoms. The study emphasises the importance of checking the interpretation of apparently straightforward terms such as "wheeze", "attacks of wheeze", "cough", and "shortness of breath". There are also implications for the design of valid questionnaires in assessing symptoms in childhood respiratory disease. The findings are important because patients and carers are increasingly recognised as having expertise that can contribute to self management of chronic illness. ${ }^{2}$

Peter Callery, RGN, RSCN, PhD Senior Lecturer School of Nursing, Midwifery and Health Visiting University of Manchester Manchester, UK

1 Cane RS, Ranganathan SC, McKenzie SA. What do parents of wheezy children understand by "wheeze"? Arch Dis Child 2000;82:327-32.

2 The expert patient: a new approach to chronic disease management for the 21st century. London: Department of Health, September 2001. http://www.doh.gov.uk/ healthinequalities. Departin 